\title{
Correction to: The feasibility of short- segment Schanz screw implanted in an oblique downward direction for the treatment of lumbar 1 burst fracture: a finite element analysis
}

\author{
Jifeng Liư ${ }^{\dagger}$, Sheng Yang ${ }^{* \dagger}$, Fei Zhou ${ }^{\dagger}$, Jianmin Lu, Chunyang Xia, Huanhuan Wang and Chao Chen
}

Correction to: J Orthop Surg Res (2020) 15:537

https://doi.org/10.1186/s13018-020-02024-7

Following publication of the original article [1], due to a typesetting error, the figure legends for Figs. 1, 3, 4, 6 and 7 were wrongly published. The correct figure legends are given below.

The authors would also like to correct the citation form page 10 .

Incorrect: $[1,19]$

Correct: [10]

The original article has been corrected.

Published online: 24 December 2020

\section{Reference}

1. Liu J, et al. The feasibility of short-segment Schanz screw implanted in an

oblique downward direction for the treatment of lumbar 1 burst fracture: a finite element analysis. J Orthop Surg Res. 2020;15:537 https://doi.org/10.

1186/s13018-020-02024-7.

The original article can be found online at https://doi.org/10.1186/s13018020-02024-7.

* Correspondence: yangsheng_88@163.com

${ }^{\dagger}$ Jifeng Liu, Sheng Yang, and Fei Zhou equally contributed to this paper and thus shared the co-first authorship.

Department of Orthopaedics, Affiliated Zhongshan Hospital of Dalian

University, 6 Jiefang Street, Zhongshan District, Dalian 116001, Liaoning,

China

(c) The Author(s). 2020 Open Access This article is licensed under a Creative Commons Attribution 4.0 International License, which permits use, sharing, adaptation, distribution and reproduction in any medium or format, as long as you give appropriate credit to the original author(s) and the source, provide a link to the Creative Commons licence, and indicate if changes were made. The images or other third party material in this article are included in the article's Creative Commons licence, unless indicated otherwise in a credit line to the material. If material is not included in the article's Creative Commons licence and your intended use is not permitted by statutory regulation or exceeds the permitted use, you will need to obtain permission directly from the copyright holder. To view a copy of this licence, visit http://creativecommons.org/licenses/by/4.0/ The Creative Commons Public Domain Dedication waiver (http://creativecommons.org/publicdomain/zero/1.0/) applies to the data made available in this article, unless otherwise stated in a credit line to the data. 

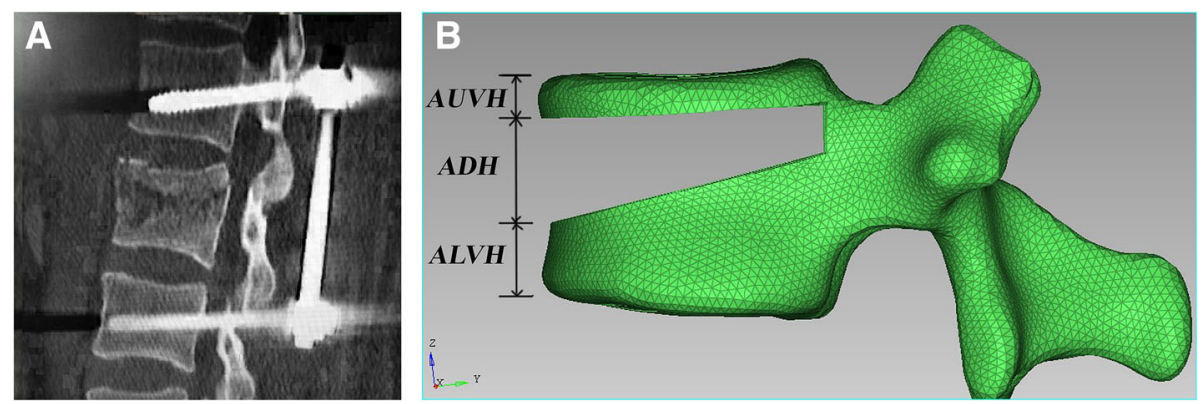

Fig. 1 a After the reduction of thoracolumbar burst fractures, the wedge-shaped bone defect area in the sagittal position was wide in the front and narrow in the back. $\mathbf{b}$ AUVH accounts for $15 \%$ of the leading edge of the vertebral body, ADH accounts for $50 \%$ of the leading edge of the vertebral body, and ALVH accounts for $35 \%$ of the leading edge of the vertebral body. AUVH, anterior upper vertebral body height above the bony defect; $\mathrm{ADH}$, anterior bony defect height; $\mathrm{ALVH}$, anterior lower vertebral body height below the bony defect 

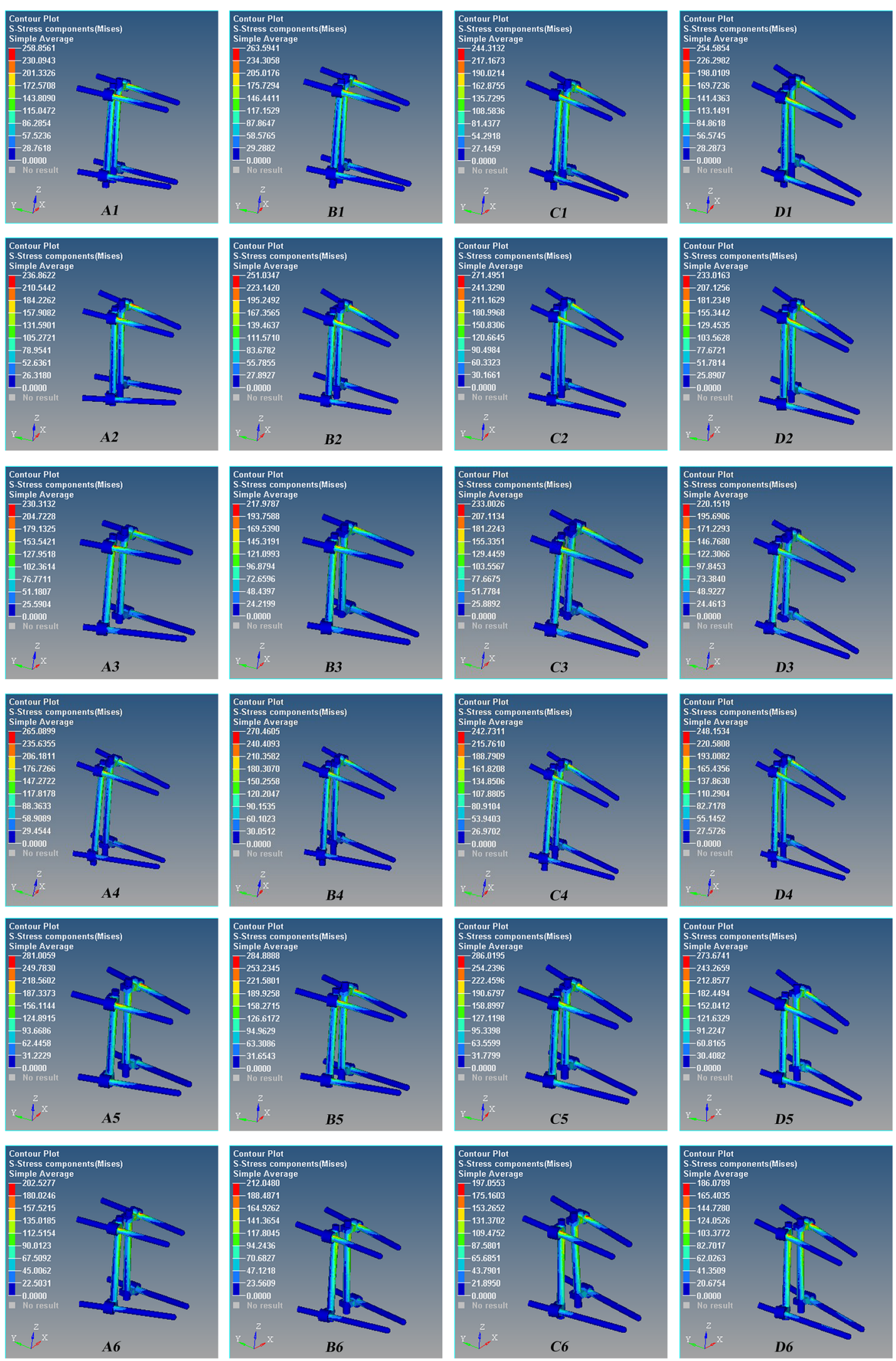

Fig. 3 The stress nephogram of the Schanz pedicle screw for the $L 1$ severe fractures after $T 12$ and $L 2$ pedicle screw fixation during anterior flexion. Red is the maximum stress. The maximum stress occurred at the interface of the proximal pedicle and cortical bone, and the stress of the upper screw is greater than that of the lower screw. A1-A6 $0^{\circ}, \mathrm{B} 1-\mathrm{B} 65^{\circ}, \mathrm{C} 1-\mathrm{C} 610^{\circ}$, and D1-D6 $15^{\circ}$. There are 6 models in each group 

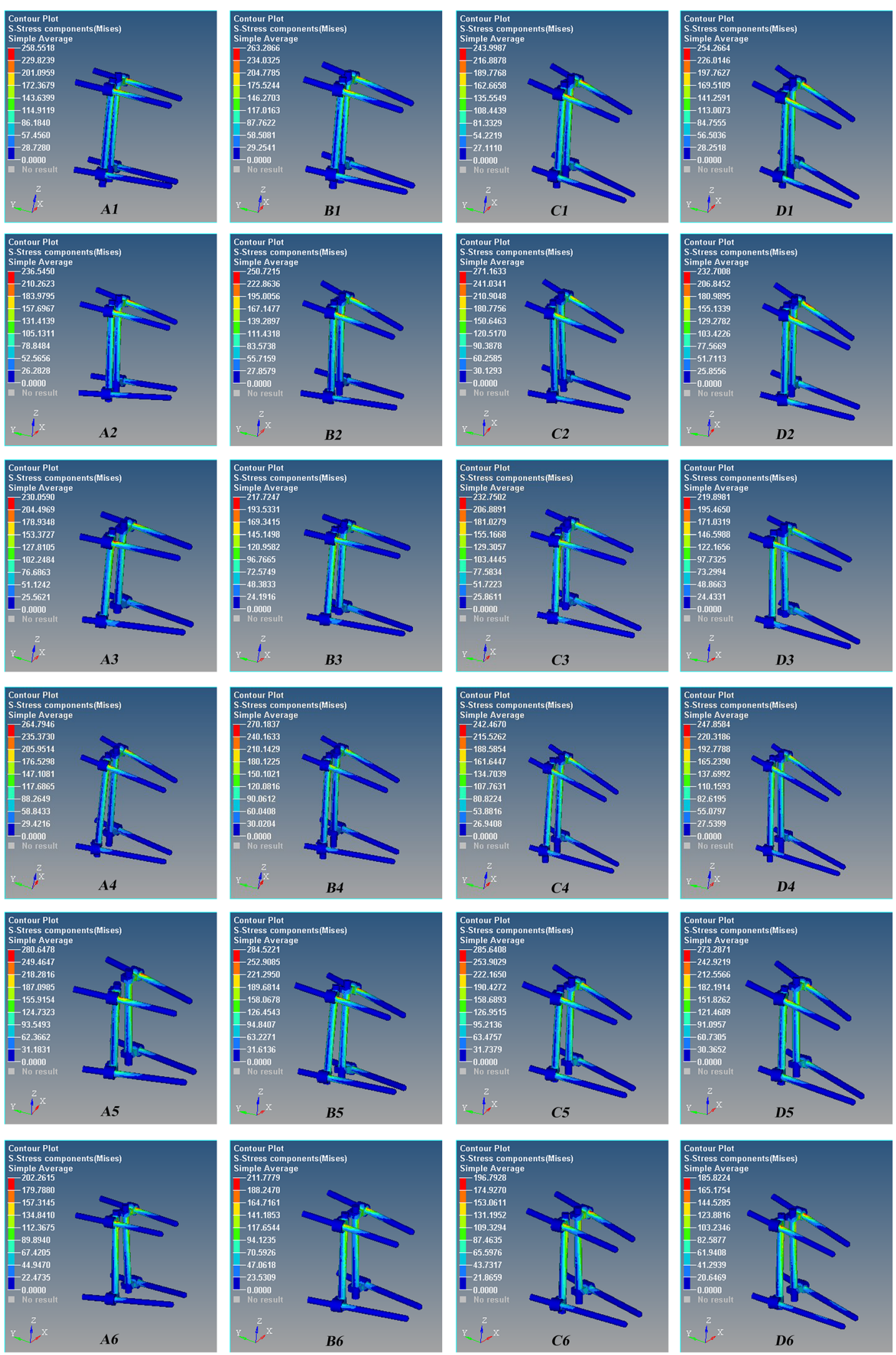

Fig. 4 The stress nephogram of the Schanz pedicle screw for the L1 severe fractures after T12 and L2 pedicle screw fixation during posterior extension. Red is the maximum stress. The maximum stress occurred at the interface of the proximal pedicle and cortical bone, and the stress of the upper screw is greater than that of the lower screw. A1-A6 $0^{\circ}, \mathrm{B} 1-\mathrm{B} 65^{\circ}, \mathrm{C} 1-\mathrm{C} 610^{\circ}$, and D1-D6 $15^{\circ}$. There are 6 models in each group 

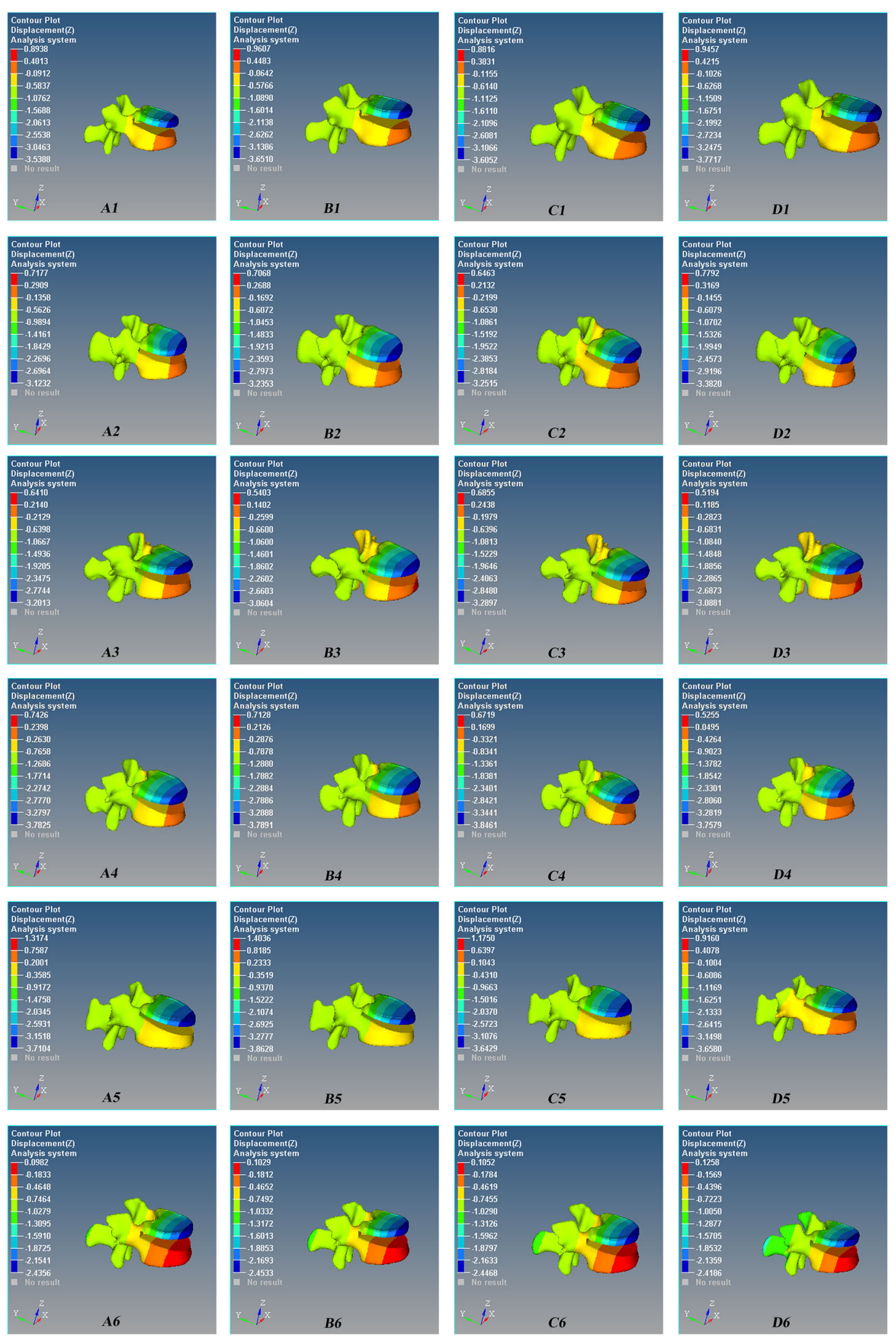

Fig. 6 Axial (Z-axis) displacement nephogram of the micro-motion of the vertebral defect area of Schanz pedicle screw for L1 severe fracture during anterior flexion. Red is the maximum displacement upward, and blue is the maximum displacement downward. A1-A6 $0^{\circ}, \mathrm{B} 1-\mathrm{B} 65^{\circ}$, C1-C6 $10^{\circ}$, and D1-D6 $15^{\circ}$. There are 6 models in each group 

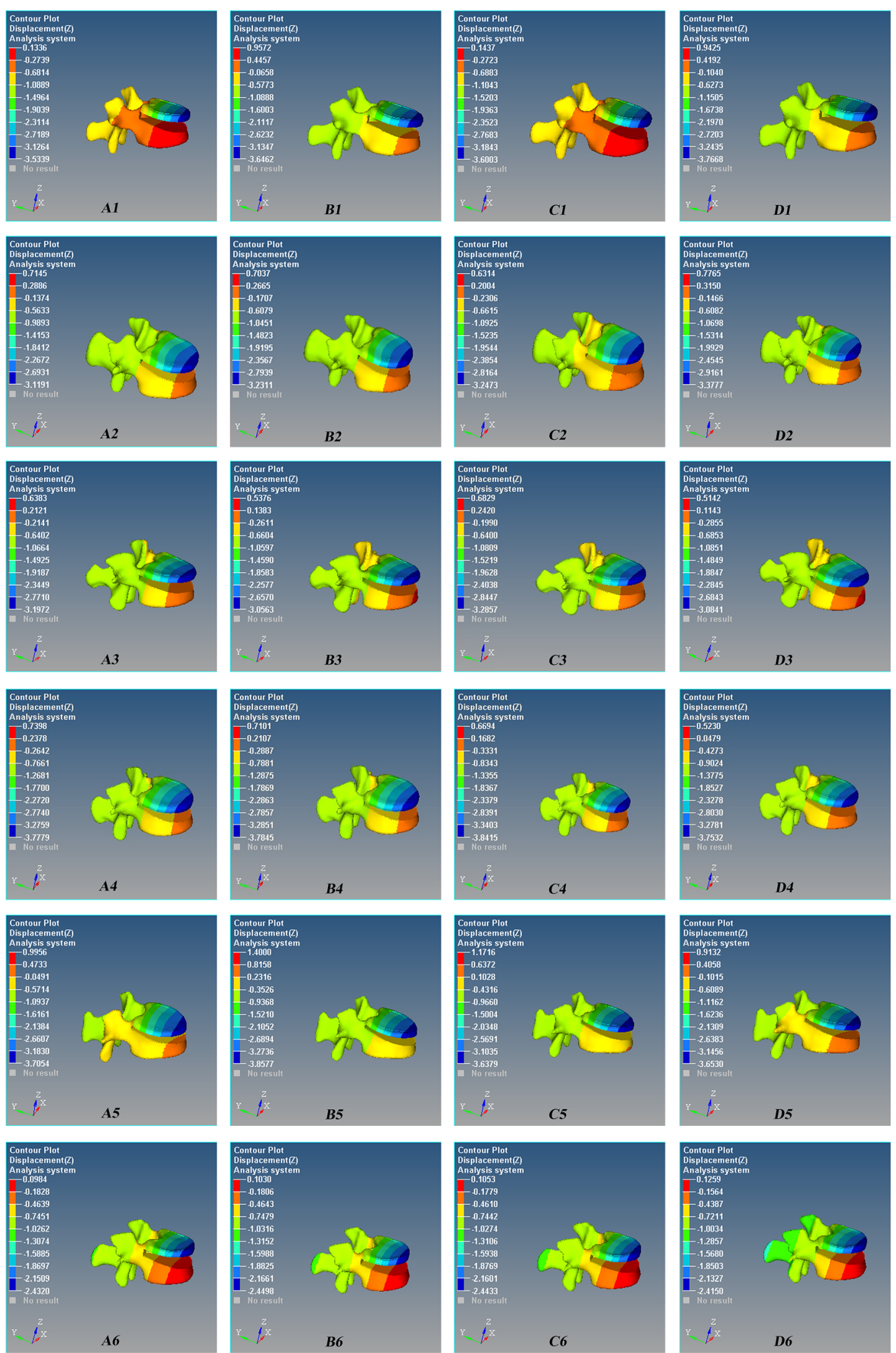

Fig. 7 Axial (Z-axis) displacement nephogram of the micro-motion of vertebral defect area of Schanz pedicle screw for L1 severe fracture during posterior extension. Red is the maximum displacement upward, and blue is the maximum displacement downward. A1-A6 $0^{\circ}, \mathrm{B} 1-\mathrm{B} 65^{\circ}, \mathrm{C} 1-\mathrm{C} 610^{\circ}$, and D1-D6 $15^{\circ}$. There are 6 models in each group 